\title{
On Spherically Symmetric Solutions of the Einstein-Maxwell Field Equations
}

\author{
Rashida Bibi ${ }^{\text {}}$, Tooba Feroze ${ }^{2}$ and Azad A. Siddiqui ${ }^{3}$ \\ 1,2,3 School of Natural Sciences, National University of Sciences and Technology, H-12, Islamabad, Pakistan. \\ ${ }^{1}$ Department of Mathematics and Statistics, International Islamic University, H-10, Islamabad, Pakistan. \\ ${ }^{1}$ rashidajahangir@yahoo.com, ${ }^{3}$ azad@sns.nust.edu.pk
}

Abstract. In literature many solution of Einstein-Maxwell's equations have been found. We consider the spherically symmetric geometry and classify the solutions of Einstein-Maxwell's equations by considering the null/non-null electromagnetic field and isotropic/anisotropic matter with the help of Segre type of spherical symmetric spacetime.

Keywords: Einstein-Maxwell Equations, null/non-null Electromagnetic Field, Segre Type, and Isotropic/Anisotropic geometry.

\section{Introduction}

A fundamental task of General Relativity is to find solutions of the Einstein field equations. In the literature, a number of solutions of these equations have been discussed [1]. The Mathematics Subject Classification MSC2010 2] gives a detail scheme to classify mathematics by subject, however in this paper we used Segre type to classify the solutions of the Einstein-Maxwell field equations. The Einstein filed equations along with the Maxwell equations are know as the Einstein-Maxwell field equations given as

$$
\begin{aligned}
& T_{\mu \nu}=R_{\mu \nu}-\frac{1}{2} R g_{\mu \nu}, \\
&\left(\sqrt{ }-g\left(F^{\mu \nu}\right)\right)_{, \nu}=j^{\mu} \sqrt{ }-g,
\end{aligned}
$$

where $T_{\mu \nu}$ is the stress energy tensor, $R_{\mu \nu}$ is the Ricci tensor, $R$ is the Ricci scalar, $g_{\mu \nu}$ is the metric tensor, $g$ is the determinate of metric tensor, $F^{\mu \nu}$ is the electromagnetic tensor, and $j^{\mu}$ is the current density.

The metric for spherically symmetric non-static space-time is given as

$$
d s^{2}=-e^{2 \nu} d t^{2}+e^{2 \lambda} d r^{2}+r^{2}\left(d \theta^{2}+\sin ^{2} \theta d \phi^{2}\right),
$$

where $\nu=\nu(t, r)$ and $\lambda=\lambda(t, r)$. While the static spherically symmetric metric is given as

$$
d s^{2}=-e^{2 \nu(r)} d t^{2}+e^{2 \lambda(r)} d r^{2}+r^{2}\left(d \theta^{2}+\sin ^{2} \theta d \phi^{2}\right) .
$$

The metric can be written in terms of null tetrad $\left(k^{a}, l^{a}, m^{a}, \bar{m}^{a}\right)[1]$.

$$
g_{a b}=2 m_{(a} \bar{m}_{b)}-2 k\left({ }_{a} l_{b}\right), k^{a} l_{b}=-1, m^{a} \bar{m}_{b}=1,
$$

\footnotetext{
${ }^{*}$ Corresponding author. Rashida Bibi ${ }^{1}$ rashidajahangir@yahoo.com
} 
where

$$
\begin{gathered}
k^{a}=1 / \sqrt{ } 2\left(-e^{-\nu} \partial / \partial t-e^{-\lambda} \partial / \partial r\right), \\
l^{a}=1 / \sqrt{ } 2\left(-e^{-\nu} \partial / \partial t+e^{-\lambda} \partial / \partial r\right), \\
m^{a}=1 / r \sqrt{ } 2\left(\partial / \partial \theta+\frac{\imath}{\sin \theta} \partial / \partial \phi\right), \\
\bar{m}^{a}=1 / r \sqrt{ } 2\left(\partial / \partial \theta-\frac{\imath}{\sin \theta} \partial / \partial \phi\right) .
\end{gathered}
$$

The trace free part of the Ricci tensor $S_{a b}=R_{a b}-\frac{1}{4} R g_{a b}$ can be written as $3 \times 3$ Hermitian matrix $\phi_{i j}(i, j=0,1,2)$

$$
\begin{array}{r}
\phi_{00}=\frac{1}{2} S_{a b} k^{a} k^{b}, \phi_{01}=\frac{1}{2} S_{a b} k^{a} m^{b}, \phi_{02}=\frac{1}{2} S_{a b} m^{a} m^{b}, \\
\phi_{11}=\frac{1}{4} S_{a b}\left(k^{a} l^{b}+m^{a} \bar{m}^{b}\right), \phi_{12}=\frac{1}{2} S_{a b} l^{a} m^{b}, \phi_{22}=\frac{1}{2} S_{a b} l^{a} l^{b} .
\end{array}
$$

If we know the Segre type of the spacetime we can find out the non-zero components of $\phi_{a b}$.

The electromagnetic tensor $F_{a b}$ is given

$$
F_{a b}=A_{a, b}-A_{b, a}
$$

where $A_{a}$ is the potential vector for the electromagnetic field. Also $F_{0 i}=E_{i}$ and $F_{i j}=c B_{i j}$, where $E_{i}$ is the electric field and $c$ is the speed of light and $B_{i j}$ is the magnetic field.

We have two cases when the trace of the stress energy tensor is zero, $T=0$ and when the trace $T \neq 0$. For the spherically symmetric geometry only non-null electromagnetic field is possible due to its spherical symmetry, where if $\mathbf{E} . \mathbf{B}=0$ and $\mathbf{E}^{2}=\mathbf{B}^{2}$, then field is null otherwise it is non-null. The stress energy tensor $T$ we choose is given by

$$
T_{j}^{i}=\operatorname{diag}\left(-\rho-\frac{E^{2}}{2}, p_{r}-\frac{E^{2}}{2}, p_{t}+\frac{E^{2}}{2}, p_{t}+\frac{E^{2}}{2}\right),
$$

where $E=E(r), p_{r}=p_{r}(r)$ is radial pressure and $p_{t}=p_{t}(r)$ is the transverse pressure. The Einstein-Maxwell filed equations for static spherically symmetric metric, are

$$
\begin{aligned}
\frac{1}{r^{2}}\left(r\left(1-e^{-2 \lambda}\right)^{\prime}\right) & =\rho+\frac{E^{2}}{2}, \\
-\frac{1}{r^{2}}\left(r\left(1-e^{-2 \lambda}\right)+\frac{2 v^{\prime}}{r} e^{-2 \lambda}\right) & =p_{r}-\frac{E^{2}}{2}, \\
e^{-2 \lambda}\left(v^{\prime \prime}+v^{\prime 2}+\frac{v^{\prime}}{r}+v^{\prime} \lambda^{\prime}-\frac{\lambda^{\prime}}{r}\right) & =p_{t}+\frac{E^{2}}{2}, \\
\sigma & =\frac{1}{r^{2}} e^{-\lambda}\left(r^{2} E\right)^{\prime} .
\end{aligned}
$$

\section{Traceless Electromagnetic Field}

For traceless filed we have $T=0$, we have

$$
e^{-2 \lambda}\left(2 \nu^{\prime \prime}+2 \nu^{\prime 2}-2 \lambda^{\prime} \nu^{\prime}+\frac{4}{r} \nu^{\prime}-\frac{4}{r} \lambda^{\prime}+\frac{2}{r^{2}}\right)-\frac{2}{r^{2}}=0
$$

By adding matter to the electromagnetic field, the speactime is classified into

1. Isotropic

2. Anisotropic 


\subsection{Isotropic Spacetime}

If the pressure is same in all directions then it is called isotropic pressure i.e. radial pressure and transverse pressure are equal, $p_{r}=p_{t}$. For isotropic case the Einstein-Maxwell field equations are

$$
\begin{aligned}
\frac{1}{r^{2}}\left(r\left(1-e^{-2 \lambda}\right)^{\prime}\right) & =\rho+\frac{E^{2}}{2}, \\
-\frac{1}{r^{2}}\left(r\left(1-e^{-2 \lambda}\right)+\frac{2 v^{\prime}}{r} e^{-2 \lambda}\right) & =p-\frac{E^{2}}{2}, \\
e^{-2 \lambda}\left(v^{\prime \prime}+v^{\prime 2}+\frac{v^{\prime}}{r}+v^{\prime} \lambda^{\prime}-\frac{\lambda^{\prime}}{r}\right) & =p+\frac{E^{2}}{2}, \\
\sigma & =\frac{1}{r^{2}} e^{-\lambda}\left(r^{2} E\right)^{\prime} .
\end{aligned}
$$

For the traceless electromagnetic field (without source) to be non-null, we have $\phi_{11}$ is the only non-zero component of the Segre tensor $S_{a b}[1,2]$. The non-zero components of $\phi_{i j}$ for the metric (3) are

$$
\begin{array}{r}
\phi_{00}=\frac{1}{2 r} e^{-2 \lambda}\left(\nu^{\prime}+\lambda^{\prime}\right), \\
\phi_{22}=\frac{1}{2 r} e^{-2 \lambda}\left(\nu^{\prime}+\lambda^{\prime}\right), \\
\phi_{11}=\frac{1}{4} e^{-2 \lambda}\left(\nu^{\prime \prime}+\nu^{\prime 2}-\nu^{\prime} \lambda^{\prime}-\frac{1}{4 r^{2}}\right)+\frac{1}{4 r^{2}} .
\end{array}
$$

Using $\phi_{11}=0$ in the Einstein-Maxwell field equations we get $\nu^{\prime}+\lambda^{\prime}=0$ which gives $p_{r}=-\rho$. Using non-null condition $\nu^{\prime}=-\lambda^{\prime}$ in $T=0$ we get

$$
r^{2}\left(e^{-2 \lambda}\right)^{\prime \prime}+4 r\left(e^{-2 \lambda}\right)^{\prime}+2 e^{-2 \lambda}-2=0
$$

which is a Cauchy-Euler Equation. Solving it we get

$$
e^{-2 \lambda}=\frac{c_{1}}{r^{2}}+\frac{c_{2}}{r}+1
$$

Using this we get the solution of the Einstein-Maxwell system

$$
\begin{gathered}
p=0, \rho=0, E^{2}=\frac{2 c_{1}}{r^{2}}, \\
\sigma=0, e^{2 \nu}=\frac{c_{1}}{r^{2}}+\frac{c_{2}}{r}+1 .
\end{gathered}
$$

So the metric in this case becomes

$$
d s^{2}=-\left(\frac{c_{1}}{r^{2}}+\frac{c_{2}}{r}+1\right) d t^{2}+\left(\frac{c_{1}}{r^{2}}+\frac{c_{2}}{r}+1\right)^{-1} d r^{2}+r^{2}\left(d \theta^{2}+\sin \theta^{2} d \phi^{2}\right),
$$

if we choose $c_{1}=Q^{2}$ and $c_{2}=-M$, where $Q$ is the charge and $M$ is the Mass then it is Reissner-Nordstrom metric, as this metric is the most general metric containing mass and charge for spherically symmetric static metric. Hence the Reissner-Nordstrom metric is the unique solution of the Einstein-Maxwell field equations for the non-null and electric field without source with isotropic matter.

\subsection{Anisotropic Spacetime}

The radial and transverse pressures are not equal in anisotropic case. Using $\nu^{\prime}=-\lambda^{\prime}$ in Eqs. 14. 15, we get

$$
p_{r}=-\rho,
$$

Till now radial pressure $p_{r}$ is taken positive but the condition of non-null electromagnetic field gives the negative radial pressure which corresponds to the dark energy. Solving further we get

$$
p_{t}=-p_{r}
$$

the tangential pressure is negative times radial pressure, which is physically not possible for the spherically static geometry also we must have $\frac{d p_{r}}{d r}<0$ and $\frac{d p_{t}}{d r}<0$ [4]. So there is no solution of the Einstein-Maxwell field equations for non-null sourceless electric field with anisotropic matter. 


\section{$3 \quad$ Electromagnetic Field With $T \neq 0$}

If the trace $T$ of the stress energy tensor is non-zero i.e. $T \neq 0$ then one needs to be careful in order to find out the relation between null/non-null field with its Segre type. Here, we are adding matter with non-null electromagnetic field. To find out the Segre type we need to write the stress energy tensor for this case and find out its Segre type. In [4] the Segre type for the non-null electromagnetic field along with the perfect fluid is discussed. If the fluid velocity vector $u$ and the null principle directions of the electromagnetic field are coplanar then the Segre type is $[1,1(11)]$ and non-zero components of $\phi_{a b}$ are $\phi_{00}=\phi_{22}$ and $\phi_{11}$ and if they are non-coplanar then Segre type is [1,111]. In our case, Segre type is $[1,1(11)]$.

\subsection{Isotropic Spacetime}

Many solutions for isotropic pressure have been obtained in literature. In this case the Einstein-Maxwell equations are

$$
\begin{aligned}
\frac{1}{r^{2}}\left(r\left(1-e^{-2 \lambda}\right)^{\prime}\right) & =\rho+\frac{E^{2}}{2}, \\
-\frac{1}{r^{2}}\left(r\left(1-e^{-2 \lambda}\right)+\frac{2 v^{\prime}}{r} e^{-2 \lambda}\right) & =p-\frac{E^{2}}{2}, \\
e^{-2 \lambda}\left(v^{\prime \prime}+v^{\prime 2}+\frac{v^{\prime}}{r}+v^{\prime} \lambda^{\prime}-\frac{\lambda^{\prime}}{r}\right) & =p+\frac{E^{2}}{2}, \\
\sigma & =\frac{1}{r^{2}} e^{-\lambda}\left(r^{2} E\right)^{\prime} .
\end{aligned}
$$

\subsection{Anisotropic Spacetime}

For anisotropic case the Einstein-Maxwell equations are [14-17]. Many solutions of the Einstein-Maxwell equations have been obtained by assuming different types of equations of state. Thirukkanesh el at. [6], Feroze [4], Mak el at. [7, J. M. Sunzu el at. [8] and M. Malaver [9] assumed linear equation of state $p_{r}=\alpha \rho-\beta$. Feroze el at. [10, 11] considered quadratic equation of state $p_{r}=\alpha \rho^{2}+\beta \rho-\gamma$, Maharaj el at. [12] also assumed quadratic equation of state of the form $p_{r}=\gamma \rho^{2}+\alpha \rho-\beta$. Mafa Takisa el at. [13] assumed the polytropic equation of state $p_{r}=k \rho^{\Gamma}$, where $\Gamma=1+\frac{1}{\eta}$ and $\eta$ is the ploytropic index. Manuel Malaver [14] assumed Van der Waals modified equation of state $p_{r}=\alpha \rho^{2}+\frac{\gamma \rho}{1+\beta \rho}$, while in [15] Manuel Malaver assumed Van der Waals modified equation of state of type $p_{r}=\alpha \rho^{\Gamma+1}+\frac{\beta \rho^{\Gamma}}{1+\gamma \rho^{Y} \Gamma}$. Thirukkanesh el at. [16] assumed Van der Waals-type equation of state $p_{r}=\alpha \rho^{2}+\frac{\gamma \rho}{1+\beta \rho}-B$. Also there are many other solutions are obtained as in $[17-20]$.

\section{Conclusion}

We can classify the electromagnetic field into two categories, traceless and with trace. when we add matter with electric field then spacetime further can be classified into isotropic and anisotropic. By taking the static spherical symmetric spacetime we checked all possible solutions of the Einstein-Maxwell equations for each case. We found out independently that for isotropic spacetime with non-null traceless electric field, the Reissner-Nordstrom metric is the unique solution of the Einstein-Maxwell field equations and there is no solution for anisotropic spacetime with non-null traceless electric field. There exists many solutions for the isotropic/ansotropic spacetime with non-null electric field with $T \neq 0$. We also discussed the Segre type of each case.

\section{References}

[1] H. Stephani, D. Kramer, M. MacCallum and C. Hoenselaers, Exact Solutions to Einstein Field Equations, (2009).

[2] Mathematics Subject Classification MSC2010. 2010. url:www.ams.org/msc/msc2010.html.

[3] C. B. G. Mclntosh, J. M. Foyster and A. W. C. Lun, The classification of the Ricci and Plebanski Tensors in General Relativity using Newman-Penrose Formalism, J. Math. Phys., 22 (1980). 
[4] Tooba Feroze. Exact solutions of the Einstein-Maxwell equations with linear equation of state, Can.J.Phys (2012). 90 1179-1183.

[5] G. S. Hall, and D. A. Negm, Physical structure of the energy-momentum tensor in General Relativity, Int. J. Theo Phys. Volume 25, (1986).

[6] S. Thirukkanesh and S. D. Maharaj, Charged Anisotropic Matter with Linear Equation of State, Class. Quant. Grav., 25 (2008).

[7] M. K. Mak and T. Harko, Quark Stars Admitting a One Parameter Group of Conformal Motions, Int. J. Mod. Phys. D, 13 (2004).

[8] J. M. Sunzu, S. D. Maharaj and S. Ray, Quark Star Model with Charged Anisotropic Matter, Astrophysics and Space Science, 354 (2014).

[9] M. Malaver, New Analytical Solutions for Quark Stars with Charged Anisotropic Matter, International Journal of Astrophysics and Space Science, 3 (2015).

[10] T. Feroze and A. A. Siddiqui, Some Exact Solutions of the Einstein-Maxwell Equations with a Quadratic Equation of State, Journal of the Korean Physical Society, 65 (2014).

[11] T. Feroze and Azad A. Siddiqui, Charged Anisoptropic Matter with Quadratic Equation of State, Gen. Rel. Grav., 43 (2011).

[12] S. D. Maharaj and P. M. Takisa, Regular Models with Quadratic Equation of State, Gen. Rel. Grav., 44 (2012).

[13] S. D. Maharaj and P. M. Takisa, Some Charged Polytropic Models, Gen. Rel. Grav., 45 (2013).

[14] M. Malaver, Regular Model for a Quark star with Van der Waals Modified Equations of State, World Applied Programming, 7 (2013).

[15] M. Malaver, Analytical Model for Charged Poltropic Stars with Van der Waals Modified Equations of Sstate, American Journal of Astronomy and Astrophysics, 1 (2013).

[16] S. Thirukkanesh and F. C. Ragel, Anisotropic Spheres with Van der Waals-type Equation of State, Pramana Journal of Physics, 83 (2014).

[17] P. M. Takisa, S. D. Maharaj and S. Ray, Stellar Objects in the Quadratic Regime, Astrophysics and Space Science, 354 (2014).

[18] M. Malaver, Polytropic Stars with Tolman IV Type Potential, AASCIT Journal of Physics, 1 (2015).

[19] M. Malaver, Strange Quark Star Model with Quadratic Equation of State, Frontiers of Mathematics and its Applications, 1 (2014).

[20] R. Shrama and B. S. Ratanpal, Relativistic Steller Model Admitting a Quadratic Equation of State, Int. J. Mod. Phys. D, 22 (2013). 